\title{
CHARACTERIZATION OF PRECOMPACT MAPS, SCHWARTZ SPACES AND NUCLEAR SPACES
}

\author{
BY \\ DAN RANDTKE
}

\begin{abstract}
A general representation theorem for "precompact" seminorms on a locally convex space is proven. Using this representation theorem the author derives a representation theorem for precompact maps from one locally convex space into another, that is analogous to the spectral representation theorem for compact maps from one Hilbert space into another and that is applicable to a very extensive class of locally convex spaces. The author uses his representation theorem to derive new characterizations of Schwartz spaces and proves analogous results for nuclear and strongly nuclear spaces.
\end{abstract}

\section{Introduction.}

1.1. The main part of this paper is devoted to a study of precompact maps from one locally convex space into another. The most important results (Theorems 2.5 and 2.18) appear in $\$ 2$.

Theorem 2.5 shows that every precompact seminorm (see Definition 2.1) on a locally convex space $E$ is "dominated" by a seminorm of the form

$$
q(x)=\sup \left|\lambda_{n}\right|\left|\left\langle x, a_{n}\right\rangle\right|,
$$

where $\lambda$ is a zero-convergent sequence of scalars and $\left\{a_{n}\right\}$ is an equicontinuous sequence in the topological dual $E^{\prime}$ of $E$. Many of the results in $\$ \S 2,3$ and 4 depend in a very essential way upon this "representation theorem."

Theorem 2.18 is our "representation theorem" for precompact linear maps from one locally convex space into another. An application of this theorem implies (see Corollary 2.19) that every compact linear map $T$ from a locally convex space $E$ into a Banach space has a representation of the form

$$
T(x)=\sum \lambda_{n}\left\langle x, a_{n}\right\rangle y_{n},
$$

where $\lambda$ belongs to $c_{0},\left\{a_{n}\right\}$ is an equicontinuous sequence in $E^{\prime}$ and $\left\{y_{n}\right\}$ is an unconditionally summable sequence in a suitable Banach space such that $\left\|\sum \xi_{n} y_{n}\right\|$ $\leqq\|\xi\|_{\infty}$, for all $\xi$ in $l_{\infty}$. $\left(\|\cdot\|_{\infty}\right.$ is the supremum norm.)

Received by the editors May 7, 1971.

AMS 1970 subject classifications. Primary 47A65, 47B05; Secondary 46A05.

Key words and phrases. Bounded map, precompact map, compact map, quasi-Schwartz map, Schwartz map, quasi-nuclear map, nuclear map, type-s map, precompact seminorm, quasi-p-nuclear seminorm, quasi-nuclear seminorm, strongly nuclear seminorm, locally convex space, Schwartz space, nuclear space, strongly nuclear space, strongly summable sequence, $\mathscr{M}$-topology, $c_{0}$-extension property. 
In $\S 2$ we introduce and study a class of precompact linear maps called quasiSchwartz maps (see Definition 2.6). Propositions 2.9 and 2.12 show that, for a very large class of locally convex spaces (which includes the class of all metrizable locally convex spaces), the precompact maps coincide with our quasi-Schwartz maps. Remark 2.13 shows that there exist precompact maps that are not quasiSchwartz. (The author can easily construct such a map using the example of Remark 2.13.) We show that the study of quasi-Schwartz maps reduces, first (see 2.11), to the study of precompact maps from one normed space into another and, finally (see $2.18,2.19$ ), to the study of a very special class of precompact endomorphisms of the Banach space $c_{0}$.

In $\S 3$ we use the results of $\S 2$ to derive new characterizations of Schwartz spaces. We then derive analogous results for nuclear and strongly nuclear spaces. The results of $\$ 3$ show that, in a certain sense, nuclear spaces are "sandwiched" between Schwartz spaces and strongly nuclear spaces.

In $\S 4$ we use Proposition 3.1 to characterize the Schwartzarity, nuclearity and strong nuclearity of the space $\mathscr{L}(E, F)$ of all continuous linear maps from one locally convex space $E$ into another $F$, where $\mathscr{L}(E, F)$ is equipped with the topology of uniform convergence on a family of bounded subsets of $E$. In the nuclear and strongly nuclear case our result is shown to be equivalent to known results; in the Schwartz space case it extends (at least, formally) a result of Grothendieck [4, Chapter I, Proposition 7.2, p. 48].

1.2. By a locally convex space, we mean a locally convex Hausdorff topological vector space over the scalar field of real or complex numbers. Let $E$ be a locally convex space. We let $E^{\prime}$ denote the topological dual of $E$. If $A$ is a subset of $E$, we let $A^{0}$ denote the polar of $A$ with respect to the dual system $\left\langle E, E^{\prime}\right\rangle$. We let $\langle x, a\rangle$ $=a(x)$ for all $x$ in $E$ and $a$ in $E^{\prime}$.

A linear map $T: E \rightarrow F$ from one locally convex space into another is precompact [respectively, compact, bounded] if $T$ maps a neighborhood of 0 in $E$ into a precompact [respectively, relatively compact, bounded] subset of $F$. ${ }^{t} T$ denotes the transpose of $T$.

1.3. Unless we indicate otherwise, all sums, sups and sequences will be taken over the positive integers.

Let $l_{p}(1 \leqq p<\infty)$ [respectively, $c_{0}, l_{\infty}$ ] denote the usual Banach space of all scalar-valued, $p$-power summable [respectively, zero-convergent, bounded] sequences. Let $s$ denote the Fréchet space of rapidly decreasing sequences (i.e. the vector space of all scalar-valued sequences $\lambda$, such that for each integer $k$

$$
q_{k}(\lambda)=\sum_{n} n^{k}\left|\lambda_{n}\right|<\infty
$$

and equipped with the topology determined by the seminorms $\left.\left\{q_{k}\right\}\right)$.

1.4. A seminorm $q$ on a locally convex space $E$ is quasi-p-nuclear $(1 \leqq p<\infty)$ 
[respectively, strongly nuclear] if there is a sequence $\lambda$ in $l_{p}$ [respectively, $\lambda$ in $s$ ] and an equicontinuous sequence $\left\{a_{n}\right\}$ in $E^{\prime}$ such that for each $x$ in $E$

$$
q(x) \leqq\left[\sum\left|\lambda_{n}\right|^{p}\left|\left\langle x, a_{n}\right\rangle\right|^{p}\right]^{1 / p}
$$

[respectively, $q(x) \leqq \sum\left|\lambda_{n}\right|\left|\left\langle x, a_{n}\right\rangle\right|$ ]. $q$ is quasi-nuclear if it is quasi-1-nuclear.

If $1 \leqq p<\infty$ is fixed, then (see [1], [2], [3,6.1, p. 184], [5, p. 275]) a locally convex space $E$ is strongly nuclear [respectively, nuclear, Schwartz] if every continuous seminorm on $E$ is strongly nuclear [respectively, quasi-p-nuclear, precompact (see Definition 2.1)].

1.5. Let $M$ be a balanced, convex, bounded subset of a locally convex space $E$. We let $E(M)=\bigcup\{\lambda M: \lambda>0\}$ and equip $E(M)$ with the norm $\|\cdot\|(M)$ defined by $\|x\|(M)=\inf \{\lambda>0: x \in \lambda M\}$. We let $J(M): E(M) \rightarrow E$ denote the identity map. (Note that $J(M)$ is always continuous.)

Let $q$ be a seminorm on a locally convex space $E$. We let $E(q)=E / q^{-1}(0)$, and we let $K(q): E \rightarrow E(q)$ denote the natural map. We equip $E(q)$ with the norm $\|\cdot\|(q)$ defined by $\|K(q) x\|(q)=q(x)$. (Note that $K(q)$ is continuous if and only if $q$ is continuous.)

Let $V$ be a balanced, convex, absorbing subset of a locally convex space $E$. Let $q$ be the gauge of $V$. We let $E(V)=E(q), K(V)=K(q)$ and $\|\cdot\|(V)=\|\cdot\|(q)$. (Note that $K(V)$ is continuous if and only if $V$ is a neighborhood of 0 in $E$.)

1.6. REMARK. Let $M$ and $N$ be balanced, convex, bounded, closed subsets of $E$ such that $N$ absorbs $M$. Let $T: E(M) \rightarrow E(N)$ and $S:\left[E^{\prime}\right]\left(N^{0}\right) \rightarrow\left[E^{\prime}\right]\left(M^{0}\right)$ be the natural maps. Let $L(N):\left[E^{\prime}\right]\left(N^{0}\right) \rightarrow[E(N)]^{\prime}$ and $L(M):\left[E^{\prime}\right]\left(M^{0}\right) \rightarrow[E(M)]^{\prime}$ be the isometric isomorphisms (into) defined via evaluation. Let $P(M): E(M) \rightarrow[E(M)]^{\prime \prime}$ and $P(N): E(N) \rightarrow[E(N)]^{\prime \prime}$ be the natural isometric isomorphisms (into) defined via evaluation. Then

(a) $\left[{ }^{t} T\right][L(N)]=[L(M)] S$,

(b) $\left[{ }^{t} S\right]\left[{ }^{t} L(M)\right][P(M)]=\left[{ }^{t} L(N)\right][P(N)] T$,

(c) $\left[{ }^{t} L(M)\right][P(M)]$ and $\left[{ }^{t} L(N)\right][P(N)]$ are isometric isomorphisms (into).

1.7. REMARK. Let $U$ and $V$ be balanced, convex, absorbing subsets of a locally convex space $E$ such that $V$ absorbs $U$. Let $T: E(U) \rightarrow E(V)$ and $S:\left[E^{\prime}\right]\left(V^{0}\right)$ $\rightarrow\left[E^{\prime}\right]\left(U^{0}\right)$ be the natural maps. Let $L(U):[E]\left(U^{0}\right) \rightarrow[E(U)]^{\prime}$ and $L(V):\left[E^{\prime}\right]\left(V^{0}\right)$ $\rightarrow[E(V)]^{\prime}$ be the natural isometric isomorphisms (into) defined via evaluation. (If $U$ and $V$ are neighborhoods of 0 in $E$, then $L(U)$ and $L(V)$ are surjective.) Let $P(U): E(U) \rightarrow[E(U)]^{\prime \prime}$ and $P(V): E(V) \rightarrow[E(V)]^{\prime \prime}$ be the natural isometric isomorphisms (into) defined via evaluation. Then

(a) $\left.{ }^{t} T\right][L(V)]=[L(U)] S$,

(b) $\left[{ }^{t} S\right]\left[{ }^{t} L(U)\right][P(U)]=\left[{ }^{t} L(V)\right][P(V)] T$.

1.8. It is known [11, Corollary 1, p. 151$]$ that if $E$ is a metrizable locally convex space, then each precompact subset of $E$ is contained in the closed, balanced, convex hull of a suitable null sequence in $E$.

1.9. By applying 1.8 it follows that if $M$ is a precompact subset of a metrizable 
locally convex space $E$, then there is a balanced, convex, bounded subset $N$ in $E$ such that $N$ absorbs $M$ and $M$ is a precompact subset of $E(N)$.

\section{Precompactness.}

2.1. Definition. A seminorm $q$ on a locally convex space $E$ is precompact if the natural map $K(q): E \rightarrow E(q)$ is precompact.

2.2. Proposition. If $q$ and $p$ are precompact seminorms on a locally convex space $E$, then $p+q$ is a precompact seminorm on $E$.

Proof. Let $T: E \rightarrow E(q) \times E(p)$ be defined by $T(x)=(K(q) x, K(p) x)$ for all $x$ in $E$. Since the kernel of $T$ equals $(q+p)^{-1}(0)$, there is a linear bijection $S: E(q+p)$ $\rightarrow T(E)$ such that $T=S[K(q+p)]$. Since

$$
\|K(q+p) x\|(q+p)=\|K(q) x\|(q)+\|K(p) x\|(p),
$$

it follows that $S$ is a topological isomorphism. Since $q$ and $p$ are precompact, $T$ is precompact. Since $S$ is a topological isomorphism (onto $T(E)$ ), the natural map $K(q+p)$ is precompact. Thus, $q+p$ is precompact.

2.3. Proposition. Let $E$ and $F$ be locally convex spaces. Let $T: E \rightarrow F$ be a continuous linear map and let $q$ be a continuous seminorm on $F$.

If either $T$ or $q$ is precompact, then $q T$ is a precompact seminorm on $E$.

Proof. Since $\|K(q)(T x)\|(q)=q(T x)=\|K(q T) x\|(q T)$ for all $x$ in $E, E(q T)$ is isometrically isomorphic to $[K(q)](T E)$ via the map $S$ defined by $S[K(q T) x]$ $=K(q)(T x)$. Therefore, $K(q T)=S^{-1}[K(q)] T$ and our result follows.

2.4. Proposition. Let $q$ be a seminorm on a locally convex space E. Let $V=\{x \in E: q(x) \leqq 1\}$. The following are equivalent:

(a) $q$ is precompact.

(b) There is a balanced, convex neighborhood $U$ of 0 in $E$ such that $V$ absorbs $U$ and the natural map $K: E(U) \rightarrow E(V)$ is precompact.

(c) There is a balanced, convex neighborhood $U$ of 0 in $E$ such that $V$ absorbs $U$ and the natural map $J:\left[E^{\prime}\right]\left(V^{0}\right) \rightarrow\left[E^{\prime}\right]\left(U^{0}\right)$ is compact.

Proof. The equivalence of (a) and (b) is easy to see.

The equivalence of (b) and (c) follows from Schauder's theorem [11, p. 111] and Remark 1.7.

2.5. THEOREM. A seminorm $q$ on a locally convex space $E$ is precompact if and only if there is a sequence $\lambda$ in $c_{0}$ and an equicontinuous sequence $\left\{a_{n}\right\}$ in $E^{\prime}$ such that for each $x$ in $E$

$$
q(x) \leqq \sup \left|\lambda_{n}\right|\left|\left\langle x, a_{n}\right\rangle\right|
$$

Proof. Suppose $q$ is precompact and let $V=\{x \in E: q(x) \leqq 1\}$. Then by (2.4) there is a balanced, convex, closed neighborhood $U$ of 0 in $E$ such that $V$ absorbs $U$ and 
the natural map $J:\left[E^{\prime}\right]\left(V^{0}\right) \rightarrow\left[E^{\prime}\right]\left(U^{0}\right)$ is compact. Therefore, $V^{0}$ is a relatively compact subset of $\left[E^{\prime}\right]\left(U^{0}\right)$; and by Remark 1.8 there is a sequence $\lambda$ in $c_{0}$ and a sequence $\left\{a_{n}\right\}$ in $U^{0}$ such that $V^{0}$ is contained in the closed, balanced, convex hull of the sequence $\left\{\lambda_{n} a_{n}\right\}$ in $\left[E^{\prime}\right]\left(U^{0}\right)$. The reader can easily verify that

$$
q(x) \leqq \sup \left|\lambda_{n}\right|\left|\left\langle x, a_{n}\right\rangle\right|
$$

for all $x$ in $E$. Therefore, the condition is necessary. We now show that it is sufficient.

Suppose that $q(x) \leqq \sup \left|\lambda_{n}\right|\left|\left\langle x, a_{n}\right\rangle\right|$, where $\lambda$ belongs to $c_{0}$ and $\left\{a_{n}\right\}$ is an equicontinuous sequence in $E^{\prime}$. Define a continuous linear map $P: E \rightarrow l_{\infty}$ by setting $P(x)=\left\{\left\langle x, a_{n}\right\rangle\right\}$. Define a continuous linear map $D: l_{\infty} \rightarrow c_{0}$ by setting $D(\xi)$ $=\left\{\lambda_{n} \xi_{n}\right\}$. Define a continuous linear map $Q: D(P E) \rightarrow E(q)$ by setting $Q(D P x)$ $=[K(q)](x)$. (Note that $\|Q\| \leqq 1$.) Since $K(q)=Q D P$ and since $D$ is precompact, it follows that $K(q)$ (and hence $q$ ) is precompact.

2.6. Definition. A linear map $T: E \rightarrow F$ from one locally convex space into another is quasi-Schwartz if there is a precompact seminorm $q$ on $E$ such that $\{T(x): q(x) \leqq 1\}$ is a bounded subset of $F$.

By Proposition 2.2 it follows that the totality of all quasi-Schwartz maps from one locally convex space into another is a vector space (when addition and scalar multiplication are defined pointwise).

2.7. Proposition. Let $E, F$ and $G$ be locally convex spaces. Let $T: E \rightarrow F$ and $S: F \rightarrow G$ be continuous linear maps.

If either $T$ or $S$ is quasi-Schwartz, then $S T$ is quasi-Schwartz.

Proof. If $T$ is quasi-Schwartz, the result follows from the fact that a continuous linear map transforms bounded sets into bounded sets.

If $S$ is quasi-Schwartz, the result follows from Proposition 2.3.

\subsection{Proposition. Every quasi-Schwartz map is precompact.}

Proof. Suppose $T: E \rightarrow F$ is quasi-Schwartz. Let $q$ be a precompact seminorm on $E$ such that $M=\{T(x): q(x) \leqq 1\}$ is bounded in $F$. Since $M$ is bounded, $q^{-1}(0)$ is contained in $\operatorname{ker} T$ and there is a continuous linear map $S: E(q) \rightarrow F$ such that $T=S[K(q)]$. Since $K(q)$ is precompact, $T$ is precompact.

2.9. Proposition. Let $T: E \rightarrow F$ be a linear map from one locally convex space into another.

If $F$ is metrizable, then $T$ is quasi-Schwartz if and only if $T$ is precompact.

Proof. Suppose $T$ is precompact, then there is a continuous seminorm $p$ on $E$ such that $M=\{T(x): p(x) \leqq 1\}$ is a precompact subset of $F$. Therefore, (Remark 1.9) there is a balanced, convex, bounded set $N$ in $F$ such that $N$ absorbs $M$ and the natural map $J: F(M) \rightarrow F(N)$ is precompact. Since $T: E \rightarrow F(M)$ is continuous, $T: E \rightarrow F(N)$ is precompact. By Proposition 2.4 the seminorm $q$ on $E$ defined by $q(x)=\|T(x)\|(N)$ is precompact. Since $\{T(x): q(x) \leqq 1\}$ is contained in $2 N, T$ is quasi-Schwartz. 
2.10. Corollary. A linear map $T: E \rightarrow F$ from a locally convex space $E$ into a normed space $F$ is precompact if and only if there is a sequence $\lambda$ in $c_{0}$ and an equicontinuous sequence $\left\{a_{n}\right\}$ in $E^{\prime}$ such that for all $x$ in $E$

$$
\|T(x)\| \leqq \sup \left|\lambda_{n}\right|\left|\left\langle x, a_{n}\right\rangle\right|
$$

2.11. Proposition. Let $T: E \rightarrow F$ be a linear map from one locally convex space into another.

$T$ is quasi-Schwartz if and only if there is a continuous seminorm $p$ on $E$, a balanced, convex, bounded subset $M$ of $F$ and a precompact linear map $S: E(p) \rightarrow F(M)$ such that $T=[J(M)] S[K(p)]$.

Proof. Suppose $T$ is quasi-Schwartz, then there is a precompact seminorm $q$ on $E$ such that $M=\{T(x): q(x) \leqq 1\}$ is bounded in $F$. Define a continuous linear map $Q: E(q) \rightarrow F(M)$ by setting $Q[K(q) x]=T(x)$. (Since $\|T(x)\|(M) \leqq q(x)$, $\|Q\| \leqq 1$.) Since $K(q): E \rightarrow E(q)$ is precompact, there is a continuous seminorm $p$ on $E$ such that $q \leqq p$ and the natural map $K: E(p) \rightarrow E(q)$ is precompact. Let $S=Q K$. Then $S: E(p) \rightarrow F(M)$ is precompact and $T=[J(M)] S[K(p)]$.

Sufficiency follows from Propositions 2.7 and 2.9.

2.12. Proposition. For a locally convex space $F$ the following are equivalent:

(a) Every precompact linear map from a locally convex space into $F$ is quasiSchwartz.

(b) Every precompact linear map from a normed space into $F$ is quasi-Schwartz.

(c) For each precompact subset $N$ of $F$, there is a balanced, convex, bounded subset $M$ of $F$ such that $M$ absorbs $N$ and $N$ is a precompact subset of $F(M)$.

(d) For each precompact subset $N$ of $F$, there is a balanced, convex, precompact subset $M$ of $F$ such that $M$ absorbs $N$ and $N$ is a precompact subset of $F(M)$.

Proof. (a) implies (b) is clear.

(b) implies (c). Let $N$ be a balanced, convex, precompact subset of $F$. Since $N$ is precompact, the natural map $J(N): F(N) \rightarrow F$ is precompact. By (b) and Proposition 2.11 there is a balanced, convex, bounded subset $M$ of $F$ and a precompact linear map $S: F(N) \rightarrow F(M)$ such that $J(N)=J(M) S$. If $x$ belongs to $N$ then $S(x)=[J(N)](x)=x$. Therefore, $S: F(N) \rightarrow F(M)$ is the natural injection and (c) follows.

(c) implies (d) is a simple consequence of 1.9.

The proof that (d) implies (a) is similar to the proof of Proposition 2.9.

2.13. REMARK. Since an uncountable product of lines does not satisfy condition (c) of Proposition 2.12, there exists a precompact map that is not quasi-Schwartz. Therefore, Definition 2.6 is justified.

In $\S 4$ (Proposition 4.3) we show that every locally convex space $F$, whose strong dual is a Schwartz space, satisfies condition (c) of Proposition 2.12. This fact, together with Propositions 2.9 and 2.12, shows that the quasi-Schwartz maps 
coincide with the precompact maps for a very extensive class of locally convex spaces. This fact also shows that there exist nonmetrizable spaces that satisfy the equivalent conditions of Proposition 2.12. Since there are no infinite dimensional normed Schwartz spaces, the equivalent conditions of Proposition 2.12 do not characterize those locally convex spaces whose strong duals are Schwartz spaces.

2.14. Definition. A sequence $\left\{y_{n}\right\}$ in a locally convex space $E$ is strongly summable if, for each $\xi$ in $l_{\infty}$, the series $\sum \xi_{n} y_{n}$ converges in $E$ and $\left\{\sum \xi_{n} y_{n}:\|\xi\|_{\infty}\right.$ $\leqq 1, \xi$ in $\left.l_{\infty}\right\}$ is bounded in $E$.

2.15. Definition. A linear map $T: E \rightarrow F$ from one locally convex space into another is a Schwartz map if there is a sequence $\lambda$ in $c_{0}$, an equicontinuous sequence $\left\{a_{n}\right\}$ in $E^{\prime}$ and a strongly' summable sequence $\left\{y_{n}\right\}$ in $F$ such that, for each $x$ in $E$,

$$
T(x)=\sum \lambda_{n}\left\langle x, a_{n}\right\rangle y_{n}
$$

2.16. REMARK. Each Schwartz map is quasi-Schwartz and the obvious analogue of Proposition 2.7 holds for Schwartz maps. If $T(x)=\sum \lambda_{n}\left\langle x, a_{n}\right\rangle y_{n}$ is a Schwartz map from one locally convex space $E$ into another $F$ (where $\lambda_{n}, a_{n}$ and $y_{n}$ satisfy the conditions of Definition 2.15) and if

$$
T_{n}(x)=\lambda_{1}\left\langle x, a_{1}\right\rangle y_{1}+\cdots+\lambda_{n}\left\langle x, a_{n}\right\rangle y_{n},
$$

then the sequence $\left\{T_{n}\right\}$ converges to $T$ in the vector space $\mathscr{L}(E, F)$ of all continuous linear maps from $E$ into $F$, when $\mathscr{L}(E, F)$ is equipped with the topology of uniform convergence on bounded subsets of $E$. Finally, the totality of all Schwartz maps from $E$ into $F$ is a vector space (when addition and scalar multiplication are defined pointwise).

2.17. Definition. A locally convex space $E$ has the $c_{0}$-extension property if every quasi-Schwartz linear map from a subspace of $c_{0}$ into $E$ can be extended to a continuous linear map from $c_{0}$ into $E$. (Note that each $\mathscr{P}_{\lambda}$-space [7, p. 2] and $c_{0}$ [8, Lemma 1(a)] have the $c_{0}$-extension property.)

2.18. THEOREM. Let $T: E \rightarrow F$ be a linear map from one locally convex space into another. If $F$ has the $c_{0}$-extension property, then the following are equivalent:

(a) $T$ is quasi-Schwartz.

(b) $T$ is Schwartz.

(c) There is a continuous linear map $P: E \rightarrow l_{\infty}$, a sequence $\lambda$ in $c_{0}$ and a continuous linear map $Q: c_{0} \rightarrow F$ such that $T=Q D_{\lambda} P$, where $D_{\lambda}: l_{\infty} \rightarrow c_{0}$ is defined by $D_{\lambda}(\xi)$ $=\left\{\lambda_{n} \xi_{n}\right\}$.

(d) Same as (c) except that $l_{\infty}$ is replaced by $c_{0}$.

Proof. (a) implies (b). Suppose $T$ is quasi-Schwartz, then there is a sequence $\lambda$ in $c_{0}$ and an equicontinuous sequence $\left\{a_{n}\right\}$ in $E^{\prime}$ such that the set

$$
M=\left\{T(x): \sup \left|\lambda_{n}\right|^{3}\left|\left\langle x, a_{n}\right\rangle\right| \leqq 1\right\}
$$

is bounded in $F$. Define a continuous linear map $P: E \rightarrow c_{0}$ by setting $P(x)$ 
$=\left\{\lambda_{n} \lambda_{n}\left\langle x, a_{n}\right\rangle\right\}$. Let $D: c_{0} \rightarrow c_{0}$ be the precompact map defined by $D(\xi)=\left\{\lambda_{n} \xi_{n}\right\}$. Define a continuous linear map $Q: D P(E) \rightarrow F(M)$ by setting $Q(D P x)=T(x)$. (Note that $\|Q\| \leqq 1$.) Let $S=[J(M)] Q D: P(E) \rightarrow F$, then $S$ is a quasi-Schwartz map such that $T=S P$. Since $F$ has the $c_{0}$-extension property $S$ has a continuous linear extension $L: c_{0} \rightarrow F$ from $c_{0}$ into $F$.

For each positive integer $n$, let $y_{n}=L\left(\lambda_{n} e_{n}\right)$, where $e_{n}=\left\{\delta_{n k}\right\}_{k}\left(\delta_{n k}=0\right.$ if $n \neq k$, $=1$ if $n=k$ ). Let $q$ be a continuous seminorm on $F$. Since $L$ is continuous, there is a constant $A>0$ such that $q(L \xi) \leqq A\|\xi\|_{\infty}$ for all $\xi$ in $c_{0}$. If $\xi$ belongs to $l_{\infty}$, then $\left\{\xi_{n} \lambda_{n}\right\}$ belongs to $c_{0}, L\left\{\xi_{n} \lambda_{n}\right\}=L\left(\sum \lambda_{n} \xi_{n} e_{n}\right)=\sum \xi_{n} y_{n}$ and $q\left(\sum \xi_{n} y_{n}\right) \leqq A\|\lambda\|_{\infty}\|\xi\|_{\infty}$. Therefore, $\left\{y_{n}\right\}$ is strongly summable. Moreover, for each $x$ in $E$,

$$
T(x)=L(P x)=L\left(\sum \lambda_{n} \lambda_{n}\left\langle x, a_{n}\right\rangle e_{n}\right)=\sum \lambda_{n}\left\langle x, a_{n}\right\rangle y_{n} .
$$

(b) implies (c). Suppose (b) holds. Define $P: E \rightarrow l_{\infty}$ by $P(x)=\left\{\left\langle x, a_{n}\right\rangle\right\}$. Define $Q: c_{0} \rightarrow F$ by $Q(\xi)=\sum \xi_{n} \lambda_{n}$. Then $P$ and $Q$ are continuous linear maps such that $T=Q D_{\lambda} P$.

(c) implies (d). Suppose (c) holds. Choose sequences $\mu$ and $\sigma$ in $c_{0}$ such that, for each $n, \lambda_{n}=\mu_{n} \sigma_{n}$. Define $L: l_{\infty} \rightarrow c_{0}$ by $L(\xi)=\left\{\xi_{n} \mu_{n}\right\}$. Define $S: c_{0} \rightarrow c_{0}$ by $S(\xi)$ $=\left\{\xi_{n} \sigma_{n}\right\}$. Then $D_{\lambda}=S L$ and (d) follows.

(d) implies (a) follows from Propositions 2.7 and 2.9.

2.19. CoRollary. Let $T: E \rightarrow F$ be a linear map from one locally convex space into another. The following are equivalent:

(a) $T$ is quasi-Schwartz.

(b) There is a locally convex space $G$, containing $F$ as a subspace, such that $T: E \rightarrow G$ is a Schwartz map.

(c) There is a sequence $\lambda$ in $c_{0}$, a continuous linear map $P: E \rightarrow c_{0}$ and a continuous linear map $Q$ from a subspace of $c_{0}$ into $F$ such that $T=Q D_{\lambda} P$, where $D_{\lambda}: c_{0} \rightarrow c_{0}$ is defined by $D_{\lambda}(\xi)=\left\{\lambda_{n} \xi_{n}\right\}$.

If $F$ is metrizable (or if $F$ satisfies one of the conditions of Proposition 2.12), then (a), (b) and (c) are equivalent to:

(d) $T$ is precompact.

(Note. If $F$ is metrizable, then $G$ in (b) can be chosen metrizable. If $F$ is normed, then $G$ can be chosen normed.)

Proof. The equivalence of (a), (b) and (c) follows from Proposition 2.18 and the fact that there is a locally convex space $G$ containing $F$ as a subspace and having the $c_{0}$-extension property. (For instance, let $G=\prod l_{\infty}\left(V^{0}\right)$, where $V$ ranges over a fundamental system of balanced, convex neighborhoods of 0 in $F$ and $l_{\infty}\left(V^{0}\right)$ denotes the Banach space of all bounded scalar-valued functions on $V^{0}$, equipped with the supremum norm; and use the well-known fact that $l_{\infty}\left(V^{0}\right)$ has the extension property $[9$, p. 21$]$.)

The second half of the corollary follows from Propositions 2.9 and 2.12 (and the above remarks concerning $G$ ). 
2.20. Corollary. For a locally convex space $F$, the following are equivalent:

(a) $F$ has the $c_{0}$-extension property.

(b) Every quasi-Schwartz linear map from a locally convex space $E$ into $F$ is a Schwartz map.

(c) Every quasi-Schwartz linear map from a subspace of $c_{0}$ into $F$ can be extended to a Schwartz map from $c_{0}$ into $F$.

If $F$ is metrizable (or if $F$ satisfies one of the conditions of Proposition 2.12), then (a), (b) and (c) above are equivalent to $\left(\mathrm{b}^{\prime}\right)$ and $\left(\mathrm{c}^{\prime}\right)$, where $\left(\mathrm{b}^{\prime}\right)$ [respectively, $\left(\mathrm{c}^{\prime}\right)$ ] is obtained from (b) [respectively, (c)] by replacing the phrase "quasi-Schwartz" with the word "precompact".

By Remark 2.16 and Corollary 2.20 it follows that every locally convex space having the $c_{0}$-extension property also has the approximation property $[11, \mathrm{p} .108]$.

\section{Schwartz spaces and nuclear spaces.}

3.1. Proposition. Let $E$ be a locally convex space.

(a) $E$ is a Schwartz space if and only if, for each continuous seminorm $q$ on $E$, there is a sequence $\lambda$ in $c_{0}$ and an equicontinuous sequence $\left\{a_{n}\right\}$ in $E^{\prime}$ such that for each $x$ in $E$

$$
q(x) \leqq \sup \left|\lambda_{n}\right|\left|\left\langle x, a_{n}\right\rangle\right| \text {. }
$$

(b) Let $0<p<\infty$. E is a nuclear space if and only if, for each continuous seminorm $q$ on $E$, there is an equicontinuous sequence $\left\{a_{n}\right\}$ in $E^{\prime}$ such that for each $x$ in $E$

$$
q(x) \leqq \sup _{n} n^{-p}\left|\left\langle x, a_{n}\right\rangle\right|
$$

(c) $E$ is a strongly nuclear space if and only if, for each continuous seminorm $q$ on $E$, there is a sequence $\lambda$ in $s$ and an equicontinuous sequence $\left\{a_{n}\right\}$ in $E^{\prime}$ such that for each $x$ in $E$

$$
q(x) \leqq \sup \left|\lambda_{n}\right|\left|\left\langle x, a_{n}\right\rangle\right| .
$$

Proof. (a) follows from Theorem 2.5.

(c) follows from the following observation. If $\lambda$ belongs to $s$ and $\left\{a_{n}\right\}$ is an equicontinuous sequence in $E^{\prime}$, then $\left\{2 n^{2} \lambda_{n}\right\}$ belongs to $s$ and, for each $x$ in $E$,

$$
\sup \left|\lambda_{n}\right|\left|\left\langle x, a_{n}\right\rangle\right| \leqq \sum\left|\lambda_{n}\right|\left|\left\langle x, a_{n}\right\rangle\right| \leqq \sup \left|2 n^{2} \lambda_{n}\right|\left|\left\langle x, a_{n}\right\rangle\right| .
$$

(b) Our proof of (b) will depend upon the following observation.

3.2. Let $0<p<\infty$. For each continuous seminorm $q$ on the space $s$ of rapidly decreasing sequences, there is an equicontinuous sequence $\left\{a_{n}\right\}$ in $s^{\prime}$ such that for each $x$ in $s$

$$
q(x) \leqq \sup n^{-p}\left|\left\langle x, a_{n}\right\rangle\right| .
$$

(Proof of 3.2). Note that if $\left\{a_{n}\right\}$ is an equicontinuous sequence in $s^{\prime}$, then for each positive integer $k,\left\{n^{k} a_{n}\right\}$ is an equicontinuous sequence in $s^{\prime}$. 
Let $q$ be a continuous seminorm on $s$. For each positive integer $n$, let $a_{n}$ in $s^{\prime}$ be the $n$th projection $\left(\left\langle x, a_{n}\right\rangle=x_{n}\right.$ for all $x$ in $\left.s\right)$ and choose a positive integer $t$ so that $q(x) \leqq \sum_{n} n^{t}\left|\left\langle x, a_{n}\right\rangle\right|$ for all $x$ in $s$; then

$$
q(x) \leqq \sup _{n} 2 n^{t+2}\left|\left\langle x, a_{n}\right\rangle\right|
$$

for all $x$ in $s$.

Let $r$ be a positive integer such that $r \geqq p$; then

$$
q(x) \leqq \sup _{n} n^{-r}\left|\left\langle x, 2 n^{t+r+2} a_{n}\right\rangle\right| \leqq \sup _{n} n^{-p}\left|\left\langle x, 2 n^{t+r+2} a_{n}\right\rangle\right|
$$

for all $x$ in $s$, where $\left\{2 n^{t+r+2} a_{n}\right\}$ is an equicontinuous sequence in $s$.

We now prove Proposition 3.1(b). Fix $0<p<\infty$ and suppose that $E$ is nuclear. By the Komura result [6] there is an index set $I$ such that $E$ is topologically isomorphic to a subspace of $s^{I}$. To complete the proof of necessity it suffices to show that each continuous seminorm on $s^{I}$ is of the desired form.

Let $q$ be a continuous seminorm on $s^{I}$. Choose $n$ elements $\nu(1), \ldots, \nu(n)$ in $I$ and choose continuous seminorms $q_{1}, \ldots, q_{n}$ on $s$ so that for each $x$ in $s^{I}$

$$
q(x) \leqq \sup \left\{q_{k}\left(x_{v(k)}\right): 1 \leqq k \leqq n\right\} .
$$

For each integer $1 \leqq k \leqq n$ use 3.2 to choose an equicontinuous sequence $\left\{a_{k r}\right\}_{r}$ in $s^{\prime}$ so that for each $x$ in $s$

$$
q_{k}(x) \leqq \sup _{r} r^{-p}\left|\left\langle x, a_{k r}\right\rangle\right| .
$$

For each $r=1,2, \ldots$ and $1 \leqq k \leqq n$, let

$$
\left\langle x, a_{(r-1) n+k}\right\rangle=n^{p}\left\langle x_{v(k)}, a_{k r}\right\rangle
$$

for all $x$ in $s^{I}$. $\left\{a_{r}\right\}$ is then an equicontinuous sequence in $\left[s^{I}\right]^{\prime}$ such that for each $x$ in $s^{I}$

$$
q(x) \leqq \sup \left\{n^{-p} r^{-p}\left|\left\langle x, a_{(r-1) n+k}\right\rangle\right|: 1 \leqq k \leqq n, r=1,2, \ldots\right\} .
$$

Since $n^{-p} r^{-p} \leqq[(r-1) n+k]^{-p}$ whenever $1 \leqq k \leqq n$ and $r=1,2, \ldots$, it follows that for each $x$ in $s^{I}$

$$
q(x) \leqq \sup _{r} r^{p}\left|\left\langle x, a_{r}\right\rangle\right|
$$

We now show that the condition in 3.1(b) is sufficient.

If the condition in 3.1(b) holds, then the following observations show that $E$ is nuclear.

If $p>1$, then each continuous seminorm $q$ on $E$ is quasi-nuclear (because $\sum n^{-p}<\infty$ and $\left.\sup n^{-p}\left|\left\langle x, a_{n}\right\rangle\right| \leqq \sum n^{-p}\left|\left\langle x, a_{n}\right\rangle\right|\right)$.

If $p \leqq 1$, then each continuous seminorm $q$ on $E$ is $(2 / p)$-nuclear (because $\sum\left(n^{-p}\right)^{2 / p}=\sum n^{-2}<\infty$ and $\left.\sup n^{-p}\left|\left\langle x, a_{n}\right\rangle\right| \leqq\left[\sum\left(n^{-p}\right)^{2 / p}\left|\left\langle x, a_{n}\right\rangle\right|^{2 / p}\right]^{p / 2}\right)$.

This completes the proof of 3.1(b). 
3.3. Proposition. For a locally convex space $E$, the following are equivalent:

(a) E is a Schwartz space.

(b) Every bounded linear map from $E$ into a locally convex space $F$ is quasiSchwartz.

(c) Every precompact linear map from $E$ into a locally convex space $F$ is quasiSchwartz.

Proof. (a) implies (b). This follows from Proposition 2.9 and the fact that if $T: E \rightarrow F$ is bounded, then there is a balanced, convex, bounded subset $M$ of $F$ and a continuous linear map $S: E \rightarrow F(M)$ such that $T=[J(M)] S$.

(b) implies (c) follows from the fact that each precompact map is bounded.

(c) implies (a). Let $T: E \rightarrow F$ be a continuous linear map from $E$ into a normed space $F$. Let $\sigma\left(F, F^{\prime}\right)$ denote the weak topology on $F$. Since each bounded subset of $F$ is $\sigma\left(E, F^{\prime}\right)$-precompact, $T$ is a precompact linear map from $E$ into $F\left[\sigma\left(F, F^{\prime}\right)\right]$. By (c) there is a precompact seminorm $q$ on $E$ such that $\{T(x): q(x) \leqq 1\}$ is a $\sigma\left(F, F^{\prime}\right)$-bounded subset of $F$. Since every $\sigma\left(F, F^{\prime}\right)$-bounded subset of $F$ is norm bounded, $T: E \rightarrow F$ is quasi-Schwartz and hence precompact. Therefore $E$ is a Schwartz space.

3.4. Remark. Analogues of Proposition 3.3 hold for nuclear and strongly nuclear spaces. We will state these analogues here.

We say that a linear map $T: E \rightarrow F$ from a locally convex space $E$ into another $F$ is quasi-nuclear [respectively, type-s], if there is a quasi-nuclear [respectively, strongly nuclear] seminorm $q$ on $E$ such that $\{T(x): q(x) \leqq 1\}$ is bounded in $F$. It is easy to see that analogues of Propositions 2.7, 2.8 and 2.11 hold for quasinuclear and type-s maps. Using these analogues it is easy to see that our definition of quasi-nuclear and type-s maps coincides with the usual definition [10, 3.2], [1], [2] in the normed space case; and that all of the important properties concerning quasi-nuclear and type-s maps in the normed space case can be lifted (via the appropriate analogue of Proposition 2.11) to the locally convex space case. By mimicking the proof of Proposition 3.3, we can prove the following analogue of Proposition 3.3.

For a locally convex space $E$ the following are equivalent:

(a) $E$ is a nuclear [respectively, strongly nuclear] space.

(b) Every bounded linear map from $E$ into a locally convex space $F$ is quasinuclear (or nuclear) [respectively, type-s].

(c) Every precompact linear map from $E$ into a locally convex space $F$ is quasinuclear (or nuclear) [respectively, type-s].

3.5. Notation. If $U$ and $V$ are balanced, convex, absorbing subsets of a locally convex space $E$, such that $U$ absorbs $V$, then for each positive integer $n$, let $\alpha_{n}(V, U)$ denote the infimum of all $\lambda>0$ such that $V \subset \lambda U+E_{n}$, where $E_{n}$ is a linear subspace of $E$ with $\operatorname{dim} E_{n} \leqq n$. 
It is well known $[10,9.4 .1]$ that if $0<p<\infty$, then a locally convex space $E$ is nuclear if and only if, for each balanced, convex neighborhood $U$ of 0 in $E$, there is another $V$, such that $U$ absorbs $V$ and, for each $n, \alpha_{n}(V, U) \leqq n^{-p}$.

By modifying the proof of $[10,9.4 .1]$ it is easy to see that a locally convex space $E$ is strongly nuclear if and only if, for each balanced, convex neighborhood $U$ of 0 in $E$, there is another $V$, such that $U$ absorbs $V$ and $\left\{\alpha_{n}(V, U)\right\} \in s$. ( $s$ is the space of rapidly decreasing sequences.)

3.6. Proposition. A locally convex space $E$ is a Schwartz space if and only if, for each balanced, convex neighborhood $U$ of 0 in $E$, there is another $V$, such that $U$ absorbs $V$ and $\left\{\alpha_{n}(V, U)\right\}$ belongs to $c_{0}$.

Proof. Suppose $E$ is a Schwartz space and let $U$ be a balanced, convex neighborhood of 0 in $E$. Since the natural map $S: E \rightarrow E(U)$ is precompact, there is a balanced, convex neighborhood $V$ of 0 in $E$ such that $S(V)$ is a precompact subset of $E(U)$. Let $\varepsilon>0$ and let $H$ be a finite subset of $E$ such that $S(V) \subset(\varepsilon / 2) S(U)$ $+S(H)$; then

$$
V \subset(\varepsilon / 2) U+H+\operatorname{ker} S \subset \varepsilon U+H .
$$

Therefore, $\alpha_{n}(V, U) \leqq \varepsilon$, where $n$ is the number of elements in $H$. Since $\alpha_{n}(V, U)$ is a decreasing function of $n$, it follows that $\left\{\alpha_{n}(V, U)\right\}$ is in $c_{0}$. We now show that the condition is sufficient.

Let $U$ be a balanced, convex neighborhood of 0 in $E$. Choose another, $V$, such that $V \subset U$ and $\left\{\alpha_{n}(V, U)\right\}$ belongs to $c_{0}$. To complete the proof, it suffices to show that $S(V)$ is a precompact subset of $E(U)$, where $S: E \rightarrow E(U)$ is the natural map.

Let $\varepsilon>0$. Let $F$ be a finite dimensional subspace of $E$ such that

$$
V \subset(\varepsilon / 2) U+F \text {. }
$$

Since $S(F \cap V)$ is an absorbing subset of $S(F)$, there is a $\lambda>0$ such that

$$
\lambda S(F \cap U) \subset S(F \cap V) .
$$

Since $V \subset U, S(F \cap V)$ is a precompact subset of $E(U)$. Therefore, there is a finite subset $H$ of $E$ such that

$$
S(F \cap V) \subset \lambda[1+(\varepsilon / 2)]^{-1}(\varepsilon / 2) S(U)+S(H) .
$$

Let $x$ belong to $V$. By (3.7) there is a $y$ in $F$ with $x-y$ in $(\varepsilon / 2) U$. Since $V \subset U$,

$$
y \in[1+(\varepsilon / 2)](U \cap F) .
$$

By (3.8) there is a $z$ in $F \cap V$ such that

$$
S\left(\lambda[1+(\varepsilon / 2)]^{-1} y-z\right)=0 .
$$

By (3.9) there is an $h$ in $H$ such that

$$
S(z-h) \in \lambda[1+(\varepsilon / 2)]^{-1}(\varepsilon / 2) S(U) .
$$


Since

$$
\begin{aligned}
S\left(x-\lambda^{-1}[1+(\varepsilon / 2)] h\right) & =S(x-y)+S\left(y-\lambda^{-1}[1+(\varepsilon / 2)] z\right)+\lambda^{-1}[1+(\varepsilon / 2)] S(z-h) \\
& \in(\varepsilon / 2) S(U)+(\varepsilon / 2) S(U)=\varepsilon S(U),
\end{aligned}
$$

it follows that

$$
S(V) \subset \varepsilon S(U)+\lambda^{-1}[1+(\varepsilon / 2)] S(H) .
$$

Therefore, $S(V)$ is a precompact subset of $S(E)=E(U)$.

4. The space $\mathscr{L}(E, F)$. Let $E$ and $F$ be locally convex spaces. Let $\mathscr{M}$ be a family of bounded subsets of $E$, such that $\bigcup \mathscr{M}$ is a total [11, p. 80] subset of $E$. Let $\mathscr{M}\left(E^{\prime}, E\right)$ denote the $\mathscr{M}$-topology $\left[11\right.$, p. 79] on $E^{\prime}$. Let $[\mathscr{L}(E, F)][\mathscr{M}]$ denote the vector space $\mathscr{L}(E, F)$ equipped with the $\mathscr{M}$-topology.

4.1. Proposition. [ $\mathscr{L}(E, F)][\mathscr{M}]$ is a Schwartz [respectively, nuclear, strongly nuclear] space if and only if $E^{\prime}\left[\mathscr{M}\left(E^{\prime}, E\right)\right]$ and $F$ are both Schwartz [respectively, nuclear, strongly nuclear] spaces.

Proof. Necessity follows from the fact that $E^{\prime}\left[\mathscr{M}\left(E^{\prime}, E\right)\right]$ and $F$ are both topologically isomorphic to subspaces of $[\mathscr{L}(E, F)][\mathscr{M}]$.

We now prove sufficiency. Without loss of generality, we may assume that each element of $\mathscr{M}$ is balanced and convex and that $\mathscr{M}$ is closed under scalar multiplication and directed by inclusion. Let $M$ belong to $\mathscr{M}$ and let $V$ be a balanced, convex neighborhood of 0 in $F$. Let

$$
(M, V)=\{T \in \mathscr{L}(E, F): T(M) \subset V\} .
$$

To complete the proof, it suffices to show that the gauge, $q$, of $(M, V)$ satisfies condition (a) [respectively, (b), (c)] in Proposition 3.1.

Let $p$ be the gauge of $M^{0}$ in $E^{\prime}$. Since $E^{\prime}\left[\mathscr{M}\left(E^{\prime}, E\right)\right]$ is Schwartz [respectively, nuclear, strongly nuclear], there is a sequence $\lambda$ in $c_{0}$ [respectively, $\lambda=\left\{1 / n^{2}\right\}, \lambda$ in $s]$ and an $\mathscr{M}\left(E^{\prime}, E\right)$-equicontinuous sequence $\left\{h_{n}\right\}$ in $\left[E^{\prime}\left[\mathscr{M}\left(E^{\prime}, E\right)\right]\right]^{\prime}$ such that for each $a$ in $E^{\prime}$

$$
p(a) \leqq \sup \left|\lambda_{n}\right|\left|\left\langle a, h_{n}\right\rangle\right| \text {. }
$$

Let $r$ be the gauge of $V$ in $F$. Since $F$ is Schwartz [respectively, nuclear, strongly nuclear], there is a sequence $\mu$ in $c_{0}$ [respectively, $\mu=\left\{1 / n^{2}\right\}, \mu$ in $s$ ] and an equicontinuous sequence $\left\{b_{k}\right\}$ in $F^{\prime}$ such that for all $y$ in $F$

$$
r(y) \leqq \sup \left|\mu_{k}\right|\left|\left\langle y, b_{k}\right\rangle\right| \text {. }
$$

Let $k$ and $n$ be positive integers. For each $T$ in $\mathscr{L}(E, F)$, let $\varphi_{k n}(T)=\left\langle b_{k} T, h_{n}\right\rangle$. $\varphi_{k n}$ is clearly a linear form on $\mathscr{L}(E, F)$. Let $U$ be a neighborhood of 0 in $F$ such that $\left\{b_{k}\right\} \subset U^{0}$. Choose $N$ in $\mathscr{M}$ so that $\left|\left\langle a, h_{m}\right\rangle\right| \leqq 1$ for all $m$ and all $a$ in $N^{0}$. If $T$ belongs to $(N, U)=\{S \in \mathscr{L}(E, F): S(N) \subset U\}$, then $\left|\varphi_{k n}(T)\right| \leqq 1$. Therefore, $\left\{\varphi_{k n}\right\}$ is an equicontinuous family of linear forms on $[\mathscr{L}(E, F)][\mathscr{M}]$. 
Let $T$ belong to $\mathscr{L}(E, F)$. Since

$$
\begin{aligned}
q(T) & =\inf \{\lambda>0: T \in \lambda(M, V)\} \\
& =\inf \{\lambda>0: T(M) \subset \lambda V\}=\sup \{r(T x): x \in M\} \\
& \leqq \sup _{k}\left[\left|\mu_{k}\right| \sup \left\{\left|\left\langle T x, b_{k}\right\rangle\right|: x \in M\right\}\right] \leqq \sup _{k}\left|\mu_{k}\right| p\left(b_{k} T\right) \\
& \leqq \sup _{k n}\left|\mu_{k}\right|\left|\lambda_{n}\right|\left|\left\langle b_{k} T, h_{n}\right\rangle\right| \leqq \sup _{k n}\left|\mu_{k}\right|\left|\lambda_{n}\right|\left|\left\langle T, \varphi_{k n}\right\rangle\right|,
\end{aligned}
$$

the following lemma implies that the gauge, $q$, of $(M, V)$ satisfies condition (a) [respectively, (b), (c)] in Proposition 3.1.

4.2. LEMMA. If $\lambda$ and $\mu$ are sequences of scalars, let $\sigma$ denote the sequence defined by

$$
\begin{aligned}
\sigma_{(n-1)(n-1)+t} & =\lambda_{t} \mu_{n} \quad \text { for } 1 \leqq t \leqq n, \\
\sigma_{(n-1)(n-1)+n+t} & =\lambda_{n} \mu_{n-t} \quad \text { for } 1 \leqq t<n .
\end{aligned}
$$

A. If $\lambda$ and $\mu$ belong to $c_{0}$, then $\sigma$ belongs to $c_{0}$.

B. If $\lambda=\mu=\left\{1 / n^{2}\right\}$, then $\left|\sigma_{n}\right| \leqq 1 / n$.

C. If $\lambda$ and $\mu$ belong to $s$, then $\sigma$ belongs to s.

Proof. The proofs of $\mathrm{A}, \mathrm{B}$ and $\mathrm{C}$ will be left to the reader.

4.3. Proposition. If each element of $\mathscr{M}$ is balanced, convex and closed and if $\mathscr{M}$ is directed by inclusion, then $E^{\prime}\left[\mathscr{M}\left(E^{\prime}, E\right)\right]$ is a Schwartz [respectively, nuclear, strongly nuclear ] space if and only if, for each $M$ in $\mathscr{M}$, there is an $N$ in $\mathscr{M}$ such that $N$ absorbs $M$ and the natural map $T: E(M) \rightarrow E(N)$ is precompact [respectively, nuclear, type-s].

Proof. It is well known [10, 4.1.2], [1], [2], [5, Proposition 3, p. 275] that $E^{\prime}\left[\mathscr{M}\left(E^{\prime}, E\right)\right]$ is a Schwartz [respectively, nuclear, strongly nuclear] space if and only if, for each $M$ in $\mathscr{M}$, there is an $N$ in $\mathscr{M}$ such that $M^{0}$ absorbs $N^{0}$ and the natural map $S:\left[E^{\prime}\right]\left(N^{0}\right) \rightarrow\left[E^{\prime}\right]\left(M^{0}\right)$ is precompact [respectively, nuclear, type-s].

Proposition 4.4 now follows from Remark 1.6 and the fact that the transpose of a precompact [respectively, nuclear, type-s] map from one normed space into another is precompact [respectively, nuclear, type-s].

Since the tensor product $E \otimes F$ of $E$ and $F$, equipped with the $\varepsilon$-topology [12, p. 434], is topologically isomorphic [12, p. 429] to a vector subspace of $\left[\mathscr{L}\left(E^{\prime}\left[\tau\left(E^{\prime}, E\right)\right], E\right)\right][e]$, where $\tau\left(E^{\prime}, E\right)$ is the Mackey topology on $E^{\prime}$ with respect to the dual system $\left\langle E, E^{\prime}\right\rangle$ and $e$ is the family of all equicontinuous subsets of $E^{\prime}$; and since $E$ and $F$ are topologically isomorphic to subspaces of $E \otimes F$, it follows that Proposition 4.1 implies the following:

4:4. Corollary. $E \otimes F$ is Schwartz [respectively, nuclear, strongly nuclear] if and only if $E$ and $F$ are both Schwartz [respectively, nuclear, strongly nuclear] spaces.

In the nuclear and strongly nuclear space cases, Corollary 4.4 is equivalent to Proposition 4.1. This follows from the following generalization of a remark by 
Pietsch [10, p. 86]: If each bounded subset of $F$ is precompact and if $F$ has the approximation property $[11, p .108]$, then the vector space $\mathscr{L}_{0}(E, F)$ of all continuous, degenerate, linear maps from $E$ into $F$ is dense in $[\mathscr{L}(E, F)][\mathscr{M}]$. Moreover, if $F$ is nuclear, then $E^{\prime}\left[\mathscr{M}\left(E^{\prime}, E\right)\right] \otimes F$ is topologically isomorphic to the vector subspace $\mathscr{L}_{0}(E, F)$ of $[\mathscr{L}(E, F)][\mathscr{M}]$.

In the nuclear space case, Pietsch $[10,5.5 .1]$ has proven Proposition 4.1 under the additional assumption that $\mathscr{M}=\beta$ (where $\beta$ denotes the family of all bounded subsets of $E$ ), and Schaefer [11, p. 173] has proven Proposition 4.1 under the additional assumptions that $E$ be semireflexive and $\mathscr{M}=\beta$.

In the nuclear space case Pietsch $[10,4.1 .6]$ has proven Proposition 4.3 under the additional assumption that $\mathscr{M}$ coincide with the family of all closed, balanced, convex bounded subsets of $E$.

In the nuclear and Schwartz space cases, Corollary 4.4 is known [4, Chapter I, p. 48], [4, Chapter II, p. 47], [10, 5.4.1], [11, p. 105].

\section{REFERENCES}

1. B. S. Brudovskií, Associated nuclear topology, mappings of type s, and strongly nuclear spaces, Dokl. Akad. Nauk SSSR 178 (1968), 271-273=Soviet Math. Dokl. 9 (1968), 61-63. MR 37 \#1952.

2. - , s-type mappings of locally convex spaces, Dokl. Akad. Nauk SSSR 180 (1968), 15-17 = Soviet Math. Dokl. 9 (1968), 572-574. MR 38 \#2561.

3. K. Floret and J. Wloka, Einführung in die Theorie der lokalkonvexen Räume, Lecture Notes in Math., no. 56, Springer-Verlag, New York, 1968. MR 37 \#1945.

4. A. Grothendieck, Produits tensoriels topologiques et espaces nucléaires, Mem. Amer. Math. Soc. No. 16 (1955). MR 17, 763.

5. J. Horváth, Topological vector spaces and distributions, vol. 1, Addison-Wesley, Reading, Mass., 1966. MR 34 \#4863.

6. T. Kōmura and Y. Kōmura, Über die Einbettung der nuklearen Räume in $(s)^{A}$, Math. Ann. 162 (1965/66), 284-288. MR 32 \#6190.

7. J. Lindenstrauss, Extension of compact operators, Mem. Amer. Math. Soc. No. 48 (1964). MR 31 \#3828.

8. J. Lindenstrauss and H. P. Rosenthal, Automorphisms in $c_{0}, l_{1}$ and $m$, Israel J. Math. 7 (1969), 227-239. MR 40 \#3273.

9. A. Persson and A. Pietsch, p-nukleare und p-integrale Abbildungen in Banachräumen, Studia Math. 33 (1969), 19-62. MR 39 \#4645.

10. A. Pietsch, Nukleare lokalkonvexe Räume, Akademie-Verlag, Berlin, 1965. MR 31 \#6114.

11. H. H. Schaefer, Topological vector spaces, Macmillan, New York, 1966. MR 33 \#1689.

12. F. Treves, Topological vector spaces, distributions and kernels, Academic Press, New York, 1967. MR 37 \#726.

Department of Mathematics, University of Georgia, Athens, Georgia 30601 